\title{
Differences in blood and urine biomarker discovery
}

\section{Opinion}

Biomarker is the measurable changes associated with the disease. Blood removes changes and urine accumulates changes because of the homeostasis mechanisms of the body. ${ }^{1}$ I believe urine is a better biomarker source than blood. This does not rule out there are biomarkers in blood. But there must be significant differences between blood and urine biomarker discovery.

First, the early and sensitive markers should be in urine because of homeostasis mechanisms. In early disease stage, the homeostasis mechanisms remain strong and effective. New changes introduced by the disease are quickly diminished and effectively removed from blood. To help maintain the stability of the blood, urine accepts many changes. By this analysis, the early and sensitive markers should be in urine instead of blood during the early stage of disease. This explains why it is so hard to find an early biomarker in blood for so many years. When biomarkers appear in blood, the disease is already in the stage when homeostasis mechanisms are impaired, changes caused by the disease can not be effectively removed from blood. Even though I am not saying it is easy to find biomarker in urine since we have not made significant progress in discover many clinically usable biomarkers, I have to propose and encourage more researchers, more companies, more funding agencies to give more support on urine biomarker discovery, after all urine is where the early markers reside.

Second, association is the key to find urine biomarker. From the definition that biomarker is the changes associated with the disease; we can see the two key words are change and association. The major reason that has been intimidating many researchers from working on urine biomarker discovery is urine changes too much even in the healthy control group. ${ }^{2}$ In urine, there are too many changes and it is easy to find many changes but hard to find which ones are associated strongly with the disease. In blood, the major difficulty is hard to find changes if there is any. Once the changes are identified, Once the changes are identified, the association is relatively easy to confirm since there is not many changes in blood anyway.

Different difficulties lead to different strategy. In urine, the confounding factors should be minimized as much as possible so that association can be identified first and then tested in clinical samples. ${ }^{3}$ In blood, the samples should be analyzed in more details since the early changes can only be found if there is a loophole in homeostasis

\author{
Volume 2 Issue 5 - 2015
}

\author{
Youhe Gao \\ Department of Biochemistry and Molecular Biology, Beijing \\ Normal University, China
}

\author{
Correspondence: Youhe Gao, Department of Biochemistry \\ and Molecular Biology, Beijing Normal University, Gene \\ Engineering and Biotechnology Beijing Key Laboratory, Beijing, \\ 100875, P. R. of China,Tel 86I058804382. \\ Email gaoyouhe@bnu.edu.cn
}

Received: October 18, 2015 | Published: October 21, 2015

mechanisms. The problem is the volume of blood can be taken for research is so limited. Nucleic acid biomarker is probably easier to be identified in blood since the amplification of nucleic acids significantly increases the detection sensitivity. Protein biomarker discovery in blood will remain a challenge before a amplification technique is developed for protein in the future. The third difference is there are more opportunities in urine early biomarker discovery because it is neglected for so many years for a wrong reason. Urine smells better.

\section{Acknowledgements}

This work was supported by the National Basic Research Program of China (2012CB517606, 2013CB530805), the Key Basic Research Program of China (No. 2013FY114100).

\section{Conflict of interest}

The author declares no conflict of interest.

\section{References}

1. Gao Y. Urine - an untapped goldmine for biomarker discovery? Sci China Life Sci. 2013;56(12):1145-1146.

2. Wu J, Gao Y. Physiological conditions can be reflected in human urine proteome and metabolome. Expert Review of Proteomics. 2015;12(6):623-636.

3. Gao Y. Opinion: Roadmap to the Urine Biomarker Era. MOJ Proteomics Bioinform. 2014;1(1):00005. 